ESAIM: PROCEEDINGS AND SURVEYS, September 2019, Vol. 66, p. 1-21

Philippe Helluy, Jean-Marc Hérard and Nicolas Seguin Editors

\title{
ADMISSIBLE EQUATIONS OF STATE FOR IMMISCIBLE AND MISCIBLE MIXTURES $*, * *$
}

\author{
Gloria FacCanoni ${ }^{1}$ AND HÉlÈne Mathis ${ }^{2}$
}

\begin{abstract}
This paper addresses the construction of admissible Equations of State (EoS) for compressible two-phase flows. We investigate two approaches. In the first one, the mixture is treated as a single fluid with a complex thermodynamic. Most of the time the available EoS are determined experimentally and are often incomplete EoS, i.e. we know only the pressure as a function of the volume and the temperature. We present here a general framework to compute a complete EoS based on such an incomplete EoS. In the second approach, each phase is depicted by its own EoS. Following the Gibbs formalism, the mixture entropy is the sum of the phasic entropies which achieves its maximum at equilibrium. Depending on the miscibility of the mixture, one gets different geometrical properties on the resulting mixture entropy. Eventually we address the coupling of mixture EoS with the dynamic of the fluid. Homogeneous Equilibrium and Relaxation Models (HEM and HRM) are introduced for an immiscible and a miscible two-phase mixture. Hyperbolicity is ensured taking advantage of the concavity properties of the mixture entropies.
\end{abstract}

Keywords Two-phase flows, equation of state, thermodynamic of equilibrium, phase transition, homogeneous equilibrium and relaxation model, hyperbolicity

Classification 2010 MCS. 76T10, 80A15, 35Q79, 35L

\section{INTRODUCTION}

Compressible multiphase flows are involved in many industrial applications, notably in nuclear framework, for instance in loss of flow or loss of coolant accidents in pressurized water reactor. For the purpose of CFD calculations, thermodynamic properties must be calculated over a wide range of states. Thus it is mandatory to provide a consistent Equation of State (EoS) to give a qualitatively and quantitatively correct material description. This EoS should represent phase equilibria over wide temperature and pressure ranges, in pure region (liquid or vapor) as well as in the VLE (Vapor-Liquid Equilibrium) region.

The construction of coherent EoS raises several difficult questions:

(1) how to obtain an accurate (and useful in CFD) description of a pure region?

(2) how to compute a given thermodynamic state by performing calculations with different couples of input variables?

* This work was partially funded by the CNRS project call NEEDS (nuclear, energy, environment, waste and society) for which the LMNC/LMNC2 projects were selected in 2014-2018.

** The second author was partially supported by the ANR project MOHYCON (ANR-17-CE40-0027-01) and would like to thank the Centre Henri Lebesgue ANR-11-LABX-0020-01 for creating an attractive mathematical environment.

${ }^{1}$ Université de Toulon, IMATH, EA 2134, avenue de l'Université, 83957 La Garde, France

2 Laboratoire de Mathématiques Jean Leray, Université de Nantes \& CNRS UMR 6629, BP 92208, F-44322 Nantes Cedex 3, France

(C) EDP Sciences, SMAI 2019

This is an Open Access article distributed under the terms of the Creative Commons Attribution License (http://creativecommons.org/licenses/by/4.0), which permits unrestricted use, distribution, and reproduction in any medium, provided the original work is properly cited. 
(3) how to describe accurately the transition from a pure region to the VLE region?

(4) CFD requires extremely fast algorithms to compute thermodynamic properties because such algorithms are frequently used in the inner iteration cycles of the process calculations. How to balance accuracy and reasonable computing times?

A classic strategy for the last question (4) is to use an analytical EoS. So the question is to construct such an analytical EoS while respecting constraints (1)-(3).

Focusing on a macroscopic description of the compressible two-phase flow, we consider that the dynamic of the fluid is depicted by an Euler-like system. The two-phase mixture is treated as a single fluid with a complex thermodynamic. ${ }^{1}$

In order to define the complex EoS, we consider two main approaches.

- The first possibility is to use an analytical EoS, valid in a wide range of temperatures and pressures. Such equations are often deduced from measurements and are usually incomplete in the sense that they define solely the pressure $p$ as a function of the volume $\tau$ and the temperature $T$. However, in the CFD calculations (based on the Euler equations), it is necessary to specify the pressure as a function of the volume and the specific internal energy $e$. To obtain these relation, an idea is to construct an entropy function $(\tau, e) \mapsto s$, which is actually a complete EoS. It is then possible to derive all incomplete EoS, including the pressure and the temperature. We present this general construction in Section 1. The section begins by the definitions of the different thermodynamic potentials both in the extensive and intensive settings. Then in paragraph 1.2 we build a complete EoS starting from the incomplete law $(\tau, T) \mapsto p$. The principle is to determine a temperature $(\tau, e) \mapsto T$ and thus an entropy $(\tau, e) \mapsto s$ thermodynamically consistent, i.e. they satisfy the first law of the Thermodynamics and a differential relation of the form $T \mathrm{~d} s=\mathrm{d} e+p \mathrm{~d} \tau$.

- The second approach we present consists in assuming that each phase is depicted by its own complete EoS (for instance its entropy). Out of equilibrium the mixture entropy is the sum of the phasic entropies. According to the second law of Thermodynamics, the mixture entropy reaches its maximum at equilibrium, under the constraints of the mass and energy conservation and a volumic constraint. Depending on the miscibility of the mixture, the latter constraint changes leading to different geometrical properties of the mixture entropy. We present in Section 2 the construction principle in the case of binary mixture. Note that it can be extended to multiphase mixture, see for instance [21,29] for three-phase framework. A particular attention is paid on the characterization of the thermodynamic equilibrium and on the concavity properties of the mixture entropy.

In Section 3, we address the coupling of the admissible EoS computed in Section 2 with the dynamic of the fluid. We present two Homogeneous Equilibrium Models (HEM) depending on the miscibility of the twophase mixture. They consist in Euler type models with the closure laws computed in Section 2. The concavity properties of the mixture entropies ensure that the models are hyperbolic. However the closure laws is often difficult to compute and may not be analytical, even for simple phasic EoS. It is then convenient to approximate the model by a relaxation procedure, see Section 4. One obtains an Homogeneous Relaxation Model (HRM), with extra equations and relaxation source terms toward the thermodynamic equilibrium, which comply with the entropy growth criterion. Again we present two models according to the miscibility of the two-phase mixture.

\section{Single FLUID THERMODYNAMiCS}

\subsection{Extensive and intensive notations}

Let consider a fluid of mass $M \geq 0$ and internal energy $E \geq 0$ occupying a volume $V \geq 0$. Let us denote $\widetilde{W} \stackrel{\text { def }}{=}(M, V)$ and $W \stackrel{\text { def }}{=}(V, M, E)$. As the fluid is homogeneous and at rest, its thermodynamic behaviour is

\footnotetext{
${ }^{1}$ Note that a way to avoid this definition of the complex EoS is to consider a two-fluid description, which treats each phase as a separated fluid depicted by balance laws of phasic mass conservation, phasic momentum and energy which are coupled through exchanging terms and nonconservative terms. Then the modelling difficulties are shifted on the definition of the exchanging terms, see $[2,17]$.
} 
entirely described by its entropy function:

$$
\begin{aligned}
S:\left(\mathbb{R}^{+}\right)^{3} & \rightarrow \mathbb{R} \\
W & \mapsto S(W) .
\end{aligned}
$$

This entropy function $S$ is uniquely and smoothly determined by the state vector $W \in\left(\mathbb{R}^{+}\right)^{3}$. Standard Thermodynamic requires that $S$ is a concave function of $W$. Then it is classic to extend it by $-\infty$ outside the close convex cone $\left(\mathbb{R}^{+}\right)^{3}$

We adopt the assumptions stated in [4] and [10]:

$$
S(W)= \begin{cases}S(W), & W \in\left(\mathbb{R}^{+}\right)^{3}, \\ -\infty, & \text { elsewhere. }\end{cases}
$$

Assumption 1. (Extensive assumptions) The entropy $S:\left(\mathbb{R}^{+}\right)^{3} \rightarrow \mathbb{R} \cup\{-\infty\}$ is such that

(i) the set of admissible states $C \stackrel{\text { def }}{=}\left\{W \in\left(\mathbb{R}^{+}\right)^{3} \mid S(W)>-\infty\right\}$ is a non-empty closed convex domain,

(ii) $S$ is a concave function of $W \in C$,

(iii) $S$ is extensive, i.e. Positively Homogeneous of degree 1 (PH1), that is

$$
\forall \lambda \in \mathbb{R}_{*}^{+}, \forall W \in C, \quad S(\lambda W)=\lambda S(W),
$$

(iv) $S$ is upper semi-continuous (u.s.c.) that is

$$
\forall W_{0} \in C, \lim _{W \rightarrow W_{0}} \sup S(W) \leq S\left(W_{0}\right)
$$

(v) $S$ is of class $\mathcal{C}^{2}$ on $C$ and its partial derivative with respect to the internal energy $E$ is strictly positive

$$
\forall W \in C, \quad \frac{\partial S}{\partial E}>0 .
$$

Assumptions (ii) and (iii) are equivalent to assume $(-S)$ sub-linear [31] or equivalently that the epigraph of $(-S)$ is a closed set in $\mathbb{R}^{3} \times \mathbb{R}$. The set $C$ is not necessarily convex, as it has been studied in [14,23]. Observe that the extensive entropy $S$ cannot be strictly concave since it is PH1.

The fundamental extensive thermodynamic relation is

$$
T \mathrm{~d} S=\mathrm{d} E+p \mathrm{~d} V-\mu \mathrm{d} M
$$

where $W \mapsto T$ is the temperature, $W \mapsto p$ the pressure and $W \mapsto \mu$ the chemical potential given by

$$
T=\left(\left.\frac{\partial S}{\partial E}\right|_{M, V}\right)^{-1}, \quad \quad p=\left.T \frac{\partial S}{\partial V}\right|_{M, E}, \quad \mu=-\left.T \frac{\partial S}{\partial M}\right|_{V, E} .
$$

As derivatives of a PH1 function, these functions are PH0 or intensive potentials. Since $S$ is PH1, it satisfies the Euler's relation $\nabla_{W} S \cdot W^{\top}=S$ which leads to another characterization of the chemical potential

$$
M \mu=-T S+p V+E .
$$

Remark 1.1. Following [9], the formula $S=S(W)$ is called the fundamental equation (or complete equation of state) of our system, and by definition contains all thermodynamic informations. An identity involving other derived quantities (such $T$ or $p$ for instance) is an incomplete equation of state, which typically does not contain all thermodynamic information. 
Remark 1.2. Additional extensive parameters can be considered to depict the thermodynamical system. For instance one takes into account the area as an additionnal extensive variable. The associated intensive parameter is then the surface tension, see [9].

\subsubsection{Intensive properties - specific description}

By homogeneity of the extensive entropy function, one may introduce the specific entropy $s$ as

$$
s \stackrel{\text { def }}{=} \frac{1}{M} S(W) \stackrel{\text { PH}}{=} S\left(\frac{W}{M}\right)=S\left(\frac{V}{M}, 1, \frac{E}{M}\right) .
$$

It corresponds to a complete EoS which is uniquely determined by the specific volume $\tau \stackrel{\text { def }}{=} V / M$ and the specific internal energy $e \stackrel{\text { def }}{=} E / M$ :

$$
\begin{aligned}
s:\left(\mathbb{R}^{+}\right)^{2} & \rightarrow \mathbb{R} \\
(\tau, e) & \mapsto s(\tau, e) .
\end{aligned}
$$

The fundamental intensive thermodynamic relation is

$$
T \mathrm{~d} s=\mathrm{d} e+p \mathrm{~d} \tau,
$$

where the temperature $T$ and the pressure $p$ are now functions of $w \stackrel{\text { def }}{=}(\tau, e)$ :

$$
w \mapsto T=\left(\left.\frac{\partial s}{\partial e}\right|_{\tau}\right)^{-1}, \quad w \mapsto p=\left.T \frac{\partial s}{\partial \tau}\right|_{e}
$$

and the chemical potential satisfies

$$
\mu=-T s+p \tau+e
$$

Assumptions 1 admit their intensive counterpart.

Assumption 2. (Intensive specific assumptions) The specific entropy $s$ is such that

- the set of admissible states $c \stackrel{\text { def }}{=}\left\{w \in\left(\mathbb{R}^{+}\right)^{2} \mid s(w)>-\infty\right\}$ is a non-empty closed convex domain,

- $s$ is of class $\mathcal{C}^{2}$ on $c$ such that

$$
T=\left(\left.\frac{\partial s}{\partial e}\right|_{\tau}\right)^{-1}>0
$$

- its Hessian matrix is negative definite, that is

$$
\left.\left.\operatorname{det}\left(H_{s}\right) \stackrel{\text { def }}{=} \frac{\partial^{2} s}{\partial \tau^{2}}\right|_{e} \frac{\partial^{2} s}{\partial e^{2}}\right|_{\tau}-\left(\frac{\partial^{2} s}{\partial \tau \partial e}\right)^{2}>0,\left.\quad \frac{\partial^{2} s}{\partial \tau^{2}}\right|_{e}<0,\left.\quad \frac{\partial^{2} s}{\partial e^{2}}\right|_{\tau}<0,
$$

which implies that $s$ is strictly concave (but the converse is wrong). ${ }^{2}$

\subsubsection{Intensive properties - volumic description}

The homogeneity of the extensive entropy allows to introduce also the volumic entropy $\sigma$ satisfying

$$
\sigma \stackrel{\text { def }}{=} \frac{1}{V} S(W) \stackrel{\text { PH}}{=} S\left(\frac{W}{V}\right)=S\left(1, \frac{M}{V}, \frac{E}{V}\right) .
$$

It is also a complete $E o S$ uniquely determined by the density $\rho \stackrel{\text { def }}{=} M / V=1 / \tau>0$ and the volumic internal energy $\varepsilon \stackrel{\text { def }}{=} E / V=\rho e$ such that

$$
\begin{aligned}
\sigma:\left(\mathbb{R}^{+}\right)^{2} & \rightarrow \mathbb{R} \\
(\rho, \varepsilon) & \mapsto \sigma(\rho, \varepsilon) .
\end{aligned}
$$

\footnotetext{
${ }^{2}$ Note that the concavity of $s$ may be deduced from the concavity of the extensive entropy $S$ and its homogeneity.
} 
As a function of $\widetilde{w} \stackrel{\text { def }}{=}(\rho, \varepsilon)$, it complies with the following total differential

$$
T \mathrm{~d} \sigma=\mathrm{d} \varepsilon-\mu \mathrm{d} \rho
$$

where the temperature $T$ and the chemical potential $\mu$ are now functions of $\widetilde{w}$ :

$$
\widetilde{w} \mapsto T=\left(\left.\frac{\partial \sigma}{\partial \varepsilon}\right|_{\rho}\right)^{-1}, \quad \widetilde{w} \mapsto \mu=-\left.T \frac{\partial \sigma}{\partial \rho}\right|_{\varepsilon},
$$

and the pressure satisfies

$$
p=T \sigma+\rho \mu-\varepsilon .
$$

Assumption 3. (Intensive volumic assumptions) The volumic entropy $\sigma$ is such that

- the set of admissible states $\widetilde{c}=\left\{\widetilde{w} \in\left(\mathbb{R}^{+}\right)^{2} \mid \sigma(\widetilde{w})>-\infty\right\}$ is a non-empty closed convex domain,

- $\sigma$ is of class $\mathcal{C}^{2}$ on $\widetilde{c}$ such that

$$
T=\left(\left.\frac{\partial \sigma}{\partial \varepsilon}\right|_{\rho}\right)^{-1}>0
$$

- its Hessian matrix is negative definite, that is

$$
\left.\left.\operatorname{det}\left(H_{\sigma}\right) \stackrel{\text { def }}{=} \frac{\partial^{2} \sigma}{\partial \rho^{2}}\right|_{\varepsilon} \frac{\partial^{2} \sigma}{\partial \varepsilon^{2}}\right|_{\rho}-\left(\frac{\partial^{2} \sigma}{\partial \rho \partial \varepsilon}\right)^{2}>0,\left.\quad \frac{\partial^{2} \sigma}{\partial \rho^{2}}\right|_{\varepsilon}<0,\left.\quad \frac{\partial^{2} \sigma}{\partial \varepsilon^{2}}\right|_{\rho}<0
$$

which implies that $\sigma$ is strictly concave (but the converse is wrong). ${ }^{3}$

\subsection{From an incomplete to a complete EoS}

It is very common to specify the EoS of a material by providing the pressure as a function of the volume and the temperature: $(\tau, T) \mapsto p$. As noted in Remark 1.1, such an expression does not embody the full range of thermodynamic informations available from the fundamental equation $S=S(W)($ or $s=s(w)$ ). However, in the CFD calculations (based on the Euler equations), one need to specify the pressure as a function of the volume and the internal energy: $(\tau, e) \mapsto p$. To do so, an idea is to construct a complete EoS in the form of a specific entropy $(\tau, e) \mapsto s$, based on the knowledge of the incomplete $\operatorname{EoS}(\tau, T) \mapsto p$. Once the complete EoS is built, all other quantities can be computed thanks to the fundamental thermodynamic relation (6) and the Maxwell relations (see appendix A).

\subsubsection{General construction}

In this section, we focus on the specific quantities and present a general method to deduce a complete EoS $(\tau, e) \mapsto s$ from an incomplete $\operatorname{EoS}(\tau, T) \mapsto p$. This construction involves determining a temperature $(\tau, e) \mapsto T$ and thus a specific entropy $(\tau, e) \mapsto s$, which are thermodynamically consistent in the sense that it complies with the fundamental relation $(6)$.

Let us consider a general incomplete $\operatorname{EoS}(\tau, T) \mapsto p$ of the form

$$
(\tau, T) \mapsto p=\frac{r T}{\tau-b}-a \mathcal{Q}(\tau)
$$

where $a$ and $b \geq 0$ are material-specific constants and $r>0$ is the universal gas constant. The choice $\mathcal{Q}(\tau)=1$ corresponds to the (Noble-Abel)-Stiffened-Gas (NASG) law [5,26,27], while the choice $\mathcal{Q}(\tau)=\tau^{-2}$ corresponds to the van der Waals law [4,25].

\footnotetext{
${ }^{3}$ Note that the concavity of $\sigma$ may be deduced from the concavity of the extensive entropy $S$ by homogeneity, or otherwise, from the concavity of the specific entropy $s$, since $\sigma=\rho s$.
} 
Since the specific entropy $s$ is a total derivative which satisfies the fundamental relation (6) (see Table 2 in Appendix A), its cross derivatives are equal:

$$
\left.\frac{\partial\left(\frac{p}{T}\right)}{\partial e}\right|_{\tau}=\left.\frac{\partial\left(\frac{1}{T}\right)}{\partial \tau}\right|_{e}
$$

Using the pressure definition (17) and the relation (18), one can exhibit an hyperbolic PDE satisfied by the temperature $(\tau, e) \mapsto T$ :

$$
\partial_{\tau} T+a \mathcal{Q}(\tau) \partial_{e} T=0
$$

Let introduce the characteristic curves $\tau \mapsto \chi(\tau)$ associated with the transport equation (19). In the $(\tau, e)$-plane they are solutions of the Cauchy problem

$$
\left\{\begin{array}{l}
\chi^{\prime}(\tau)=a \mathcal{Q}(\tau) \\
\chi\left(\tau_{0}\right)=\chi_{0}
\end{array}\right.
$$

that is

$$
\chi(\tau)=\chi_{0}+a \int_{\tau_{0}}^{\tau} \mathcal{Q}(x) \mathrm{d} x .
$$

The temperature $\mathcal{T} \stackrel{\text { def }}{=} T(\tau, e=\chi(\tau))$ is constant along the characteristic curves, hence it holds $\mathcal{T}(\tau)=\mathcal{T}\left(\tau_{0}\right)$ and yields

$$
T(\tau, e)=T\left(\tau_{0}, e-a \int_{\tau_{0}}^{\tau} \mathcal{Q}(x) \mathrm{d} x\right) .
$$

For illustrative purposes, we choose the temperature as

$$
T(\tau, e)=\frac{e-a \int_{\tau_{0}}^{\tau} \mathcal{Q}(\tau) \mathrm{d} \tau}{c_{v}},
$$

with $c_{v}$ the specific heat (which is assumed constant here for sake of simplicity). Finally a complete EoS can be obtained by integrating the fundamental relation (6)

$$
\mathrm{d} s=\frac{p}{T} \mathrm{~d} \tau+\frac{1}{T} \mathrm{~d} e=\left(\frac{c_{v}}{e-a \int \mathcal{Q}(\tau) \mathrm{d} \tau} p(\tau, e)\right) \mathrm{d} \tau+\frac{c_{v}}{e-a \int \mathcal{Q}(\tau) \mathrm{d} \tau} \mathrm{d} e .
$$

Using (8) and treating $\tau$ as a constant, by integrating the relation (24) with respect to $e$ gives

$$
s(\tau, e)=\int \frac{1}{T} \mathrm{~d} e+\omega(\tau)=\int \frac{c_{v}}{e-a \int \mathcal{Q}(\tau) \mathrm{d} \tau} \mathrm{d} e+\omega(\tau)=c_{v} \ln \left(e-a \int \mathcal{Q}(\tau) \mathrm{d} \tau\right)+\omega(\tau) .
$$

In order to determine the function $\omega$, one has to derive (25) with respect to $\tau$. It holds

$$
\left.\frac{\partial s}{\partial \tau}\right|_{e}=\frac{p}{T}=\frac{r}{\tau-b}-\frac{c_{v}}{e-a \int \mathcal{Q}(\tau) \mathrm{d} \tau} a \mathcal{Q}(\tau)
$$

Using the general form of the pressure (17) and the temperature definition (23), by identification, the function $\omega(\tau)$ satisfies $\omega^{\prime}(\tau)=\frac{r}{\tau-b}$ and consequently $\omega(\tau)=r \ln (\tau-b)$. Finally the specific entropy reads

$$
s(\tau, e)=c_{v} \ln \left(e-a \int \mathcal{Q}(\tau) \mathrm{d} \tau\right)+r \ln (\tau-b)+s_{0},
$$

where $s_{0}$ is a numerical constant of integration. 
We underline that, given $\tau \mapsto \mathcal{Q}(\tau)$, the definition of the temperature $T$ is not unique (and, as a consequence, the specific entropy is not unique as well).

\subsubsection{Applications}

Considering the aforementioned choices of $\mathcal{Q}(\tau)$, one obtains the following complete EoS:

- the Noble-Abel-Stiffened-Gas law corresponds to $\mathcal{Q}(\tau)=1$ which leads to

$$
s(\tau, e)=c_{v} \ln (e+a \tau+\text { const. })+r \ln (\tau-b)+s_{0} .
$$

Getting const. $=-q+a b$ where $q$ is a constant, we recover the Noble-Abel-Stiffened-Gas EoS described in $[5,26,27]$. This specific entropy $s$ is a strictly concave function of $(\tau, e)$ and complies with Assumptions 2.

- The van der Waals law is obtained setting $\mathcal{Q}(\tau)=\tau^{-2}$ and gives

$$
s(\tau, e)=c_{v} \ln \left(e+\frac{a}{\tau}+\text { const. }\right)+r \ln (\tau-b)+s_{0} .
$$

Classically const. $=0$, see $[4,25]$. However $(\tau, e) \mapsto s$ is not concave everywhere. Thus the van der Waals specific entropy fits into the foregoing framework (Assumption 2) only if we restrict attention to regions where $(\tau, e) \mapsto s$ is concave. If we apply the well-know Maxwell area construction, which is equivalent to replace $s$ by its concave hull (the smallest concave function greater that or equal to $s$ in some region), the graph contains a line and this geometrical property is related to the physical interpretation of phase transition (see [4]). In [15,28] the authors investigate the link between the Legendre Transform (LT) and the sup-convolution and the concave hull. The sup-convolution is transformed into an addition by the LT and applying twice a LT to any function is an elegant way to compute its concave hull. A fast LT algorithm is proposed in [15] in order to compute tabulated Equation of State of mixture with application to the van der Waals law.

\section{Computing Admissible EOS FOR two-Phase miXture}

We consider an ideally isolated system of fixed mass $M \geq 0$, volume $V \geq 0$ and internal energy $E \geq 0$, composed of two phases $A$ and $B$ which are entirely described by their entropy functions $S_{k}$, function of their masses $0 \leq M_{k} \leq M$, volumes $0 \leq V_{k} \leq V$ and internal energies $0 \leq E_{k} \leq E, k \in \mathbb{K} \stackrel{\text { def }}{=}\{A, B\}$. In the following we denote $W_{k} \stackrel{\text { def }}{=}\left(V_{k}, M_{k}, E_{k}\right)$ and $\widetilde{W_{k}} \stackrel{\text { def }}{=}\left(M_{k}, E_{k}\right)$.

Mass and energy conservations ensure that

$$
\begin{gathered}
M=M_{A}+M_{B}, \\
E=E_{A}+E_{B} .
\end{gathered}
$$

Depending on the miscibility of the mixture, the volume constraint should differ:

- as the two phases are immiscible, they are separated by an interface, leading to

$$
V=V_{A}+V_{B},
$$

so that $W=W_{A}+W_{B}$;

- when the two phases are miscible, we assume they are completely intimate and no interface arises, meaning that they behave like ideal gases in the sense that

$$
V=V_{A}=V_{B},
$$

so that $\widetilde{W}=\widetilde{W}_{A}+\widetilde{W}_{B}$. 
Remark 2.1. Note that in both cases, we assume that no vacuum appears in the model. Otherwise one should consider that $V \geq V_{A}+V_{B}$. Besides we also assume that there is no interaction at the molecular level between the two different components. Indeed if one considers the mixture of 1 liter of water and 1 liter of pure alcohol (at atmospheric pressure), then the resulting total volume $V$ is less than 2 liters. Neither assumptions (29) or (30) are valid; one should consider $V<V_{A}+V_{B}$.

Remark 2.2. As the two phases are immiscible, one should consider $V_{A}+V_{B} \leq V$. As explained in [16], if the phasic pressures $p_{k}, k \in \mathbb{K}$, are always strictly positive, then the constraint is necessarily saturated and boils down to $V=V_{A}+V_{B}$. In a perfectly miscible case, one should consider $V_{A} \leq V$ and $V_{B} \leq V$. Again as the phasic pressures and the temperatures are non-negative, the constraint is saturated in the sense that $V=V_{A}=V_{B}$.

\subsection{Characterization of the thermodynamic equilibrium}

Since the fluid system is ideally isolated, the second law of Thermodynamic states that all internal processes lead to an increase on the total extensive entropy of the system. When the extensive entropy reaches its maximum value, the system is at thermodynamic equilibrium. In this section we address the definition of the extensive equilibrium entropy of the mixture depending on its miscibility.

Out of equilibrium, the mixture entropy $\mathscr{S}$ is the sum of the phasic entropies $S_{k}$. For $\left(W_{A}, W_{B}\right) \in C_{A} \times C_{B}$, it reads

$$
\mathscr{S}\left(W_{A}, W_{B}\right)=S_{A}\left(W_{A}\right)+S_{B}\left(W_{B}\right)=S_{A}\left(V_{A}, M_{A}, E_{A}\right)+S_{B}\left(V_{B}, M_{B}, E_{B}\right)
$$

Definition 2.3. (Extensive equilibrium entropy of an immiscible mixture) Let $W \stackrel{\text { def }}{=}(V, M, E) \in\left(\mathbb{R}^{+}\right)^{3}$ be the state vector of the two-phase immiscible mixture. The equilibrium entropy is:

$$
S^{\mathrm{immi}}(W) \stackrel{\text { def }}{=} \max _{\left(W_{A}, W_{B}\right) \in \Omega^{\mathrm{immi}}} \mathscr{S}\left(W_{A}, W_{B}\right)
$$

where

$$
\begin{aligned}
\Omega^{\text {immi def }} & \left.\stackrel{(}{=}\left(W_{A}, W_{B}\right) \in C_{A} \times C_{B} \mid(27),(28) \text { and (29) hold }\right\} \\
& =\left\{\left(W_{A}, W_{B}\right) \in C_{A} \times C_{B} \mid W=W_{A}+W_{B}\right\} .
\end{aligned}
$$

Definition 2.4. (Extensive equilibrium entropy of a miscible mixture) Let $V=V_{0}>0$ fixed. Let $\widetilde{W} \stackrel{\text { def }}{=}(M, E) \in\left(\mathbb{R}^{+}\right)^{2}$ be the state vector of the two-phase miscible mixture. The equilibrium entropy is

$$
S^{\mathrm{mi}}\left(V_{0}, \widetilde{W}\right) \stackrel{\text { def }}{=} \underset{\left(\widetilde{W}_{A}, \widetilde{W}_{B}\right) \in \Omega^{\mathrm{mi}}}{\operatorname{Sax}} \mathscr{S}\left(\left(V_{0}, \widetilde{W}_{A}\right),\left(V_{0}, \widetilde{W}_{B}\right)\right)
$$

where

$$
\begin{aligned}
\Omega^{\mathrm{mi}} \stackrel{\text { def }}{=}\left\{\left(\widetilde{W}_{A}, \widetilde{W}_{B}\right) \in \widetilde{C}_{A} \times \widetilde{C}_{B} \mid(27) \text { and (28) hold }\right\} \\
=\left\{\left(\widetilde{W}_{A}, \widetilde{W}_{B}\right) \in \widetilde{C}_{A} \times \widetilde{C}_{B} \mid \widetilde{W}=\widetilde{W}_{A}+\widetilde{W}_{B}\right\} .
\end{aligned}
$$

Remark 2.5. In [16] the authors highlight that the maximization processes (32) and (33) are called supconvolution operations. This operation is well-known in convex analysis, see for instance [31], where it is denoted $\square$. It reads

$$
\begin{aligned}
S^{\mathrm{immi}}(W) & =S_{A} \square S_{B}(W)=\sup _{W_{A} \in C_{A}} S_{A}\left(W_{A}\right)+S_{B}\left(W-W_{A}\right), \\
S^{\mathrm{mi}}\left(V_{0}, \widetilde{W}\right) & =S_{A} \square_{V_{0}} S_{B}\left(V_{0}, \widetilde{W}\right)=\sup _{\widetilde{W}_{A} \in \widetilde{C}_{A}} S_{A}\left(V_{0}, \widetilde{W_{A}}\right)+S_{B}\left(V_{0}, \widetilde{W}-\widetilde{W}_{A}\right) .
\end{aligned}
$$


Note that in the miscible case, the sup-convolution is partial and acts only on the mass and energy variables, the volume $V_{0}$ being fixed. The sup-convolution shares many properties. Among them, it ensures the existence and the concavity of the equilibrium mixture entropies.

Remark 2.6. If one assumes that no mass transfer arises between the two phases, then the phasic masses are fixed. In the case of an immiscible mixture without phase transition, the mixture entropy is also concave with respect to $V, M, E$ and $M_{A}$ as soon as the phasic entropies $S_{k}$ are sub-linear, see [23]. As miscible mixture without phase transition is concerned, the mixture entropy is also a concave function of $(V, M, E)$ since it corresponds to a linear transformation of a concave function.

Following [23], one can prove that the extensive entropies $S^{\text {immi }}, S^{\mathrm{mi}}: C \rightarrow \mathbb{R} \cup\{-\infty\}$ are PH1 concave functions of $W \in C$, where $C=C_{A}+C_{B}$ is a non empty convex cone.

When the equilibrium entropies $S^{\lambda}, \lambda=$ immi or mi, are differentiable with respect to the volume $V$, the mass $M$ and the energy $E$, one can define the mixture temperature, pressure and chemical potential at equilibrium as follows:

$$
T \stackrel{\text { def }}{=}\left(\left.\frac{\partial S^{\lambda}}{\partial E}\right|_{M, V}(W)\right)^{-1},\left.\quad \quad \quad \stackrel{\text { def }}{=} T \frac{\partial S^{\lambda}}{\partial V}\right|_{M, E}(W),\left.\quad \mu \stackrel{\text { def }}{=} T \frac{\partial S^{\lambda}}{\partial M}\right|_{V, E}(W),
$$

leading to the extensive fundamental relation

$$
T \mathrm{~d} S^{\lambda}=\mathrm{d} E+p \mathrm{~d} V-\mu \mathrm{d} M .
$$

We now turn to the characterization of the equilibrium. In the definition of the mixture entropy, the maximizer may be not unique but the upper value is unique. Besides if the phasic entropies $S_{k}$ are upper semicontinuous, then the supremum is actually a maximum.

Proposition 2.7. When the maximum is reached in the interior of the set of constraints, one has:

- the maximum is always characterized by the thermal equilibrium

$$
T=T_{A}=T_{B}
$$

- the maximum defining $S^{i m m i}$ in the immiscible case is characterized by

$$
p=p_{A}=p_{B},
$$

while, in the miscible case with $S^{m i}$, it corresponds to the Dalton's law [30]:

$$
p=p_{A}+p_{B}
$$

- as phase transition occurs in the miscible and immiscible mixture, the equilibrium is also characterized by

$$
\mu=\mu_{A}=\mu_{B} .
$$

We refer to $[1,16,22]$ for a proof for the immiscible case and to [15] for both miscible and immiscible cases. Here we focus on the equilibrium reached in the interior of the domain of $S^{\lambda}$. It can also be reached on the boundary of the set of constraints. In that case, one should characterized the maximum by inequalities on the pressures and temperatures. Since the immiscible case is treated in [23] (considering the mass $M_{A}$ is fixed), we just give some aspects in the miscible case, that is $V=V_{A}=V_{B}=V_{0}>0$ fixed. If the maximum is reached at the point $(\bar{M}, \bar{E})$, the concavity of the entropy leads to

$$
\nabla_{\left(M_{A}, E_{A}\right)}\left(S_{A}\left(V_{0}, M_{A}, E_{A}\right)+S_{B}\left(V_{0}, M-M_{A}, E-E_{A}\right)\right)\left(\left(M_{A}, E_{A}\right)-(\bar{M}, \bar{E})\right) \leq 0 .
$$

Assume that the energy constraints is saturated, then one has 
- either $\bar{E}=E$ and $\frac{1}{T_{A}}-\frac{1}{T_{B}} \geq 0$;

- or $\bar{E}=0$ and $\frac{1}{T_{A}}-\frac{1}{T_{B}} \leq 0$.

Conversely, if the mass constraint is saturated, one gets

- either $\bar{M}=M$ and $-\frac{\mu_{A}}{T_{A}}+\frac{\mu_{B}}{T_{B}} \geq 0$;

- or $\bar{M}=0$ and $-\frac{\mu_{A}}{T_{A}}+\frac{\mu_{B}}{T_{B}} \leq 0$.

\subsection{Intensive entropies and geometrical interpretation}

We now turn to the definition of intensive quantities. The system is now entirely described by its intensive specific entropy $s$ defined by (5) as a function of $w \stackrel{\text { def }}{=}(\tau, e) \in\left(\mathbb{R}^{+}\right)^{2}$ or by its intensive volumic entropy $\sigma$ defined by $(11)$ as a function of $\widetilde{w} \stackrel{\text { def }}{=}(\rho, \varepsilon) \in\left(\mathbb{R}^{+}\right)^{2}$. We assume that they comply with Assumptions 2 and 3 respectively.

Let us introduce the volume fraction $\alpha_{k}$, the mass fraction $\varphi_{k}$ and the energy fraction $z_{k}$ of the phase $k \in \mathbb{K}$ defined respectively by

$$
\alpha_{k} \stackrel{\text { def }}{=} \frac{V_{k}}{V}, \quad \varphi_{k} \stackrel{\text { def }}{=} \frac{M_{k}}{M}, \quad z_{k} \stackrel{\text { def }}{=} \frac{E_{k}}{E},
$$

which belong to $[0,1]$.

- Each phase $k \in \mathbb{K}$ is depicted by its specific phasic entropy $s_{k}$ defined by

$$
s_{k}\left(\tau_{k}, e_{k}\right) \stackrel{\text { def }}{=} S_{k}\left(\tau_{k}, 1, e_{k}\right)
$$

It is a function of the specific volume $\tau_{k}$ and a specific internal energy $e_{k}$ such that

$$
\tau_{k}=\frac{V_{k}}{M_{k}}=\frac{\alpha_{k}}{\varphi_{k}} \tau, \quad e_{k}=\frac{E_{k}}{M_{k}}=\frac{z_{k}}{\varphi_{k}} e,
$$

satisfying the phasic fundamental relation

$$
T_{k} \mathrm{~d} s_{k}=\mathrm{d} e_{k}+p_{k} \mathrm{~d} \tau_{k}
$$

- Eventually the phase $k \in \mathbb{K}$ may be depicted by its volumic phasic entropy $\sigma_{k}$ defined by

$$
\sigma_{k}\left(\rho_{k}, \varepsilon_{k}\right) \stackrel{\text { def }}{=} S_{k}\left(1, \rho_{k}, \varepsilon_{k}\right)
$$

It is a function of the density $\rho_{k}$ and the volumic internal energy $\varepsilon_{k}$ such that

$$
\rho_{k}=\frac{M_{k}}{V_{k}}=\frac{\varphi_{k}}{\alpha_{k}} \rho=\frac{1}{\tau_{k}}, \quad \varepsilon_{k}=\frac{E_{k}}{V_{k}}=\frac{z_{k}}{\alpha_{k}} \varepsilon=\frac{z_{k}}{\alpha_{k}} \rho e=\frac{e_{k}}{\tau_{k}},
$$

satisfying the phasic fundamental relation

$$
T_{k} \mathrm{~d} \sigma_{k}=\mathrm{d} \varepsilon_{k}-\mu_{k} \mathrm{~d} \rho_{k}
$$

The extensive constraints (27)-(30) admit their intensive counterparts. The mass and energy conservations (27) and (28) give the following constraints on the fractions:

$$
\begin{aligned}
& 1=\varphi_{A}+\varphi_{B}, \\
& 1=z_{A}+z_{B} .
\end{aligned}
$$


- In the case of an immiscible mixture, the extensive volume constraint (29) translates into

$$
1=\alpha_{A}+\alpha_{B}
$$

and, associated to (43), leads to

$$
\begin{aligned}
& \tau=\varphi_{A} \tau_{A}+\varphi_{B} \tau_{B}, \\
& e=\varphi_{A} e_{A}+\varphi_{B} e_{B} .
\end{aligned}
$$

Out of equilibrium, the extensive mixture entropy (31) can be written in intensive specific formulation using the homogeneity property of the extensive entropies $S_{k}, k \in \mathbb{K}$ :

$$
s\left(w_{A}, w_{B}\right) \stackrel{\text { def }}{=} \frac{\mathscr{S}}{M}=\varphi_{A} s_{A}\left(w_{A}\right)+\varphi_{B} s_{B}\left(w_{B}\right) .
$$

- In the miscible case, the extensive volume constraint (30) translates into

$$
1=\alpha_{A}=\alpha_{B}
$$

and, associated to (46), we have

$$
\begin{aligned}
& \rho=\rho_{A}+\rho_{B}, \\
& \varepsilon=\varepsilon_{A}+\varepsilon_{B} .
\end{aligned}
$$

Out of equilibrium, the extensive mixture entropy (31) can be written in intensive volumic formulation using the homogeneity property of the extensive entropies $S_{k}, k \in \mathbb{K}$ :

$$
\sigma\left(\widetilde{w}_{A}, \widetilde{w}_{B}\right) \stackrel{\text { def }}{=} \frac{\mathscr{S}}{V}=\sigma_{A}\left(\widetilde{w}_{A}\right)+\sigma_{B}\left(\widetilde{w}_{B}\right)
$$

We now turn to the description of the intensive mixture entropy at equilibrium. The optimization processes (32) and (33) can be derived in an intensive formulation as follows, leading to an elegant geometrical interpretation.

- As the immiscible case is considered, at equilibrium, the optimization problem (32) reads under its intensive formulation

$$
s^{\mathrm{immi}}(\tau, e)=\max _{\substack{1=\varphi_{A}+\varphi_{B} \\ w=\varphi_{A} w_{A}+\varphi_{B} w_{B}}}\left(s\left(w_{A}, w_{B}\right)\right),
$$

under constraints (48), (51) and (52). One observes that the formulation (58) defines nothing else but the concave hull of the maximum of the two partial entropies $s_{k}, k \in \mathbb{K}$ :

$$
s^{\text {immi }}(w)=\text { concave hull of } \max \left(s_{A}(w), s_{B}(w)\right)
$$

Therefore the mixture entropy is concave but not strictly concave. In a region corresponding to the vaporliquid equilibrium, the graph of $s^{\mathrm{immi}}$ contains a line of plane. This characterization is highlighted for instance in $[1,3,11,12,15,16]$.

- In the miscible case, the total volume $V$ is fixed and equal to $V_{0}>0$. Then the optimization process allows to define the volumic mixture entropy as

$$
\sigma^{\operatorname{mi}}(\widetilde{w})=\max _{\widetilde{w}=\widetilde{w}_{A}+\widetilde{w}_{B}}\left(\sigma\left(\widetilde{w}_{A}, \widetilde{w}_{B}\right)\right)
$$


under the mass and energy conservation constraints (55) and (56). Observe that the intensive mixture entropy remains a sup-convolution operation of the phasic entropies

$$
\sigma^{\mathrm{mi}}(\rho, \varepsilon)=\sigma_{A} \square \sigma_{B}(\rho, \varepsilon),
$$

see Remark 2.5. The properties of the sup-convolution operation ensure that if the phasic entropies are strictly concave, then the mixture entropy is strictly concave as well [18,31]. Note that the thermodynamic equilibrium is still characterized by Proposition 2.7 that is the equilibrium of the temperature, the chemical potential and the Dalton's law on the pressures.

The equilibrium entropies properties are stated in the following statement:

Proposition 2.8. Consider the thermodynamic equilibrium with the equilibrium temperature $T$ and the equilibrium chemical potential satisfying (36) and (39), i.e. $T=T_{A}=T_{B}$ and $\mu=\mu_{A}=\mu_{B}$.

- If the mixture is immiscible, the equilibrium pressure $p$ satisfies (37) and the intensive equilibrium entropy $s^{i m m i}$ is a concave function of $(\tau, e)$ and satisfies

$$
T \mathrm{~d} s^{i m m i}=\mathrm{d} e+p \mathrm{~d} \tau .
$$

- If the mixture is miscible, the equilibrium pressure $p$ complies with the Dalton's law (38) $p=p_{A}+p_{B}$ and the volumic equilibrium entropy of the mixture is a concave function of $(\rho, \varepsilon)$ and satisfies

$$
T \mathrm{~d} \sigma^{m i}=\mathrm{d} \varepsilon-\mu \mathrm{d} \rho .
$$

Proof.

- We multiply the phasic Gibbs relations (44) $T_{k} \mathrm{~d} s_{k}=\mathrm{d} e_{k}+p_{k} \mathrm{~d} \tau_{k}$ by the mass fractions $\varphi_{k}$ and we sum over $k \in \mathbb{K}$ :

$$
\begin{aligned}
0 & =\sum_{k \in \mathbb{K}}\left(\varphi_{k} T_{k} \mathrm{~d} s_{k}-\varphi_{k} \mathrm{~d} e_{k}-\varphi_{k} p_{k} \mathrm{~d} \tau_{k}\right) \stackrel{(36)(37)}{=} T \sum_{k \in \mathbb{K}}\left(\varphi_{k} \mathrm{~d} s_{k}\right)-\sum_{k \in \mathbb{K}}\left(\varphi_{k} \mathrm{~d} e_{k}\right)-p \sum_{k \in \mathbb{K}}\left(\varphi_{k} \mathrm{~d} \tau_{k}\right) \\
& =T \sum_{k \in \mathbb{K}} \mathrm{d}\left(\varphi_{k} s_{k}\right)-\sum_{k \in \mathbb{K}} \mathrm{d}\left(\varphi_{k} e_{k}\right)-p \sum_{k \in \mathbb{K}} \mathrm{d}\left(\varphi_{k} \tau_{k}\right)-\sum_{k \in \mathbb{K}}\left(T s_{k}-e-p \tau_{k}\right) \mathrm{d} \varphi_{k} \\
& =T \mathrm{~d} s^{\mathrm{immi}}-\mathrm{d} e-p \mathrm{~d} \tau+\sum_{k \in \mathbb{K}} \mu_{k} \mathrm{~d} \varphi_{k} \stackrel{(39)}{=} T \mathrm{~d} s^{\mathrm{immi}}-\mathrm{d} e-p \mathrm{~d} \tau+\mu \mathrm{d}\left(\sum_{k \in \mathbb{K}} \varphi_{k}\right) \\
& =T \mathrm{~d} s^{\mathrm{immi}}-\mathrm{d} e-p \mathrm{~d} \tau+\mu \mathrm{d}(1)=T \mathrm{~d} s^{\mathrm{immi}}-\mathrm{d} e-p \mathrm{~d} \tau .
\end{aligned}
$$

- We sum the phasic relations (47) for $k \in \mathbb{K}$ : since the temperatures are equal, see (36), as well as the chemical potentials, see (39), then

$$
0=\sum_{k \in \mathbb{K}}\left(T_{k} \mathrm{~d} \sigma_{k}-\mathrm{d} \varepsilon_{k}-\mu_{k} \mathrm{~d} \rho_{k}\right) \stackrel{(36)(39)}{=} T \mathrm{~d} \sigma^{\mathrm{mi}}-\mathrm{d} \varepsilon-\mu \mathrm{d} \rho,
$$

and

$$
\begin{aligned}
\sum_{k \in \mathbb{K}} p_{k} & =\sum_{k \in \mathbb{K}}\left(\rho_{k} \mu_{k}+T_{k} \sigma_{k}-\varepsilon_{k}\right) \\
& \stackrel{(36)(39)}{=} \mu\left(\sum_{k \in \mathbb{K}} \rho_{k}\right)+T\left(\sum_{k \in \mathbb{K}} \sigma_{k}\right)-\left(\sum_{k \in \mathbb{K}} \varepsilon_{k}\right) \stackrel{(55)(56)(57)}{=} \mu \rho+T \sigma-\varepsilon=p
\end{aligned}
$$




\section{Homogeneous Equilibrium for two-Phase Flows}

We now take into account the dynamic of the two-phase mixture assuming that the two-phases have the same velocity. The aim of this section is to provide an Homogeneous Equilibrium Euler's system, called HEM model. The HEM model has to be endowed with appropriate closure laws in agreement with the thermodynamic equilibria studied in Section 2. Emphasis is given to the hyperbolicity and the entropy structure of the models. For the sake of simplicity we consider mono-dimensional case.

\subsection{Immiscible case}

At thermodynamic equilibrium the immiscible two-phase flow is depicted by the multicomponent Euler system

$$
\left\{\begin{array}{l}
\partial_{t} \rho+\partial_{x}(\rho u)=0, \\
\partial_{t}(\rho u)+\partial_{x}\left(\rho u^{2}+p^{\mathrm{immi}}\right)=0, \\
\partial_{t}(\rho \mathcal{E})+\partial_{x}\left(\left(\rho \mathcal{E}+p^{\mathrm{immi}}\right) u\right)=0, \\
\mathcal{E}=\frac{1}{2} u^{2}+e, \\
\forall k \in \mathbb{K}: p_{k}=p_{k}\left(\tau_{k}, e_{k}\right), \tau_{k}=\rho_{k}^{-1},
\end{array}\right.
$$

where the flow has a density $\rho$ (we also define the specific volume $\tau=1 / \rho$ ), a velocity $u$, a pressure $p^{\text {immi, }}$ and an internal energy $e$ ( $\mathcal{E}$ being the total energy). The phase $k \in \mathbb{K}$ is depicted by its mass fraction $\varphi_{k}$, its pressure $p_{k}$, its specific volume $\tau_{k}$ and its specific internal energy $e_{k}$, see Section 2.2. All the phases evolve at the same velocity $u$.

The multicomponent Euler system admits eight equations and has thirteen unknowns which are

$$
\left\{\rho, u, \mathcal{E}, p^{\mathrm{immi}}, e, \varphi_{A}, \varphi_{B}, \tau_{A}, \tau_{B}, e_{A}, e_{B}, p_{A}, p_{B}\right\}
$$

Thus one has to provide five closure laws. The first two closure laws are given by the constraints (51) and (52). The three remaining closure laws are given by Proposition 2.7, that is

$$
\left\{\begin{array}{l}
T^{\mathrm{immi}}=T_{A}=T_{B}, \\
p^{\mathrm{immi}}=p_{A}=p_{B}, \\
\mu=\mu_{A}=\mu_{B}
\end{array}\right.
$$

where $T^{\mathrm{immi}}$ and $p^{\mathrm{immi}}$ are the thermodynamic temperature and pressure of the two-phase flow and $p^{\mathrm{immi}}=$ $p^{\mathrm{immi}}(1 / \rho, e)$.

To prove the hyperbolicity of the system, one would like to apply the Godunov-Mock theorem using the function $\eta:(\tau, u, \mathcal{E}) \mapsto-s^{\mathrm{immi}}\left(\tau, \mathcal{E}-u^{2} / 2\right)$ as a Lax entropy of the system, see for instance [6,24,29]. Unfortunately, since the equilibrium entropy $s^{i m m i}$ is not a strictly concave function of its arguments, this argument does not apply in our case. One has to study the eigenvalues of the system and to prove that the speed of sound of the mixture is real. First notice that, for any state vector $w \stackrel{\text { def }}{=}(\tau, e)$, the speed of sound of the system can be written as

$$
c^{2}(w)=-\tau^{2} T(w)(p(w),-1) H_{s^{\mathrm{immi}}}(w)\left(\begin{array}{c}
p(w) \\
-1
\end{array}\right),
$$

where $H_{s}^{\mathrm{immi}}(w)$ denotes the Hessian matrix of $s^{\mathrm{immi}}$. Because $s^{\mathrm{immi}}$ is the concave hull of the phasic entropies $s_{k}, k \in \mathbb{K}$, it follows that

- for all $w$ in pure phase regions

$$
v^{\top} H_{s^{\mathrm{immi}}}(w) v<0, \quad \forall v \neq 0,
$$


- for all $w$ in the VLE region

$$
\text { it exists a unique } v(w) \neq 0 \text {, such that }(v(w))^{\top} H_{s^{\text {immi }}}(w) v(w)=0 \text {. }
$$

The hyperbolicity results is stated in $[1,11,12]$ by proving the following theorem:

Theorem 3.1. For any state vector $w$ in the VLE region,

$$
v(w) \not \equiv(p(w),-1),
$$

so that the system (64) is strictly hyperbolic.

\subsection{Miscible case}

We now turn to the miscible case. At thermodynamic equilibrium the miscible two-phase flow is depicted by the multicomponent Euler system

$$
\left\{\begin{array}{l}
\partial_{t} \rho+\partial_{x}(\rho u)=0 \\
\partial_{t}(\rho u)+\partial_{x}\left(\rho u^{2}+p^{\mathrm{mi}}\right)=0 \\
\partial_{t}(\rho \mathcal{E})+\partial_{x}\left(\left(\rho \mathcal{E}+p^{\mathrm{mi}}\right) u\right)=0 \\
\mathcal{E}=\frac{1}{2} u^{2}+\frac{\varepsilon}{\rho} \\
\forall k \in \mathbb{K}: p_{k}=p_{k}\left(\rho_{k}, \varepsilon_{k}\right)
\end{array}\right.
$$

The system (70) admits six equations and thirteen unknowns which are

$$
\left\{\rho, u, \mathcal{E}, p^{\mathrm{mi}}, \varphi_{A}, \varphi_{B}, \tau_{A}, \tau_{B}, e_{A}, e_{B}, p_{A}, p_{B}\right\}
$$

Hence one has to provide seven closure laws. The first two closure laws are given by the constraints (55) and (56). The characterization of the equilibrium provides the five remaining closure laws:

$$
\left\{\begin{array}{l}
T^{\mathrm{mi}}=T_{A}=T_{B}, \\
p^{\mathrm{mi}}=p_{A}+p_{B}, \\
\mu^{\mathrm{mi}}=\mu_{A}=\mu_{B},
\end{array}\right.
$$

where $p^{\mathrm{mi}}$ is the pressure of the two-phase flow and $p^{\mathrm{mi}}=p^{\mathrm{mi}}(\rho, \varepsilon)$.

The hyperbolicity of the system (70) relies on its entropy structure. Indeed in Section 2.2 we have shown that the volumic equilibrium entropy is strictly concave as soon as the phasic entropies are. In this case the Godunov-Mock theorem applies (see for instance [6,24,29]) and we have the following theorem:

Theorem 3.2. The strictly convex function $\eta(\rho, u, \mathcal{E}) \mapsto-\sigma\left(\rho, \rho \mathcal{E}-\rho u^{2} / 2\right)$ is a Lax entropy for the system $(70)$ associated to the entropy flux $f_{\eta}(\rho, u, \mathcal{E})=-u \sigma$. Thus the system (70) is strictly hyperbolic.

\section{Homogeneous Relaxation TWO-PHASE MODELS}

The equilibrium two-phase models presented in the previous section are difficult to use for practical computations. Indeed the determination of the equilibrium mixture pressure law may be tedious, and even worse, it is not analytical when considering standard phasic EoS such as stiffened gas law, see for instance [11, 12, 28]. In [15] the authors propose to use a Fast Legendre Transform algorithm to compute admissible mixture pressure laws (see also Remark 2.5). Again the resulting mixture pressure law is not analytical but solely a tabulated one. 
In order to avoid the manipulation of a complex pressure law, it is convenient to approximate the equilibrium Euler system by a Homogeneous Relaxation Model (HRM), following [3,11-13,16, 19, 20]. It consists in adding convection equations of additional variables and to modify the pressure to make it depend on these variables. Relaxation source terms complete the new convection equations. The relaxation structure should be consistent with the second law of Thermodynamics. In particular, the source terms have to comply with the entropy growth criterion and bring the system to thermodynamic equilibrium.

\subsection{Immiscible case}

Following $[3,11,19]$, the additional variables we consider are the fractions of mass, volume and energy of the phase $A: Y \stackrel{\text { def }}{=}\left(\varphi_{A}, \alpha_{A}, z_{A}\right)$. Note that one may have chosen another set of variables as well. When no transfer occurs between the liquid and the vapor, the fractions should be perfectly convected, that is

$$
\partial_{t} Y+u \partial_{x} Y=0
$$

Combining with the conservation of total mass and adding the source terms to the fraction equations gives

$$
\left\{\begin{array}{l}
\partial_{t}(\rho Y)+\partial_{x}(\rho u Y)=Q \\
\partial_{t} \rho+\partial_{x}(\rho u)=0 \\
\partial_{t}(\rho u)+\partial_{x}\left(\rho u^{2}+p\right)=0 \\
\partial_{t}(\rho \mathcal{E})+\partial_{x}((\rho \mathcal{E}+p) u)=0
\end{array}\right.
$$

with the closure law

$$
p=p\left(1 / \rho, e, \varphi_{A}, \alpha_{A}, z_{A}\right) .
$$

The system (73) admits the concave function

$$
s:\left(\tau, e, \varphi_{A}, \alpha_{A}, z_{A}\right) \mapsto \varphi_{A} s_{A}\left(\frac{\alpha_{A}}{\varphi_{A}} \tau, \frac{z_{A}}{\varphi_{A}} e\right)+\varphi_{B} s_{B}\left(\frac{1-\alpha_{A}}{1-\varphi_{A}} \tau, \frac{1-z_{A}}{1-\varphi_{A}} e\right),
$$

already defined in (53), as an entropy function. Recall that its concavity is deduced from the concavity of the phasic entropies $s_{k}, k \in \mathbb{K}$. Moreover it complies with

$$
\mathrm{d} s=\sum_{k \in \mathbb{K}} \frac{z_{k}}{T_{k}} \mathrm{~d} e+\sum_{k \in \mathbb{K}} \alpha_{k} \frac{p_{k}}{T_{k}} \mathrm{~d} \tau+\sum_{k \in \mathbb{K}} \partial_{\varphi_{k}} s \mathrm{~d} \varphi_{k}+\sum_{k \in \mathbb{K}} \partial_{\alpha_{k}} s \mathrm{~d} \alpha_{k}+\sum_{k \in \mathbb{K}} \partial_{z_{k}} s \mathrm{~d} z_{k} .
$$

We can then identify the mixture pressure (74) and the mixture temperature by

$$
\frac{p\left(1 / \tau, e, \varphi_{A}, \alpha_{A}, z_{A}\right)}{T\left(1 / \tau, e, \varphi_{A}, \alpha_{A}, z_{A}\right)}=\sum_{k \in \mathbb{K}} \alpha_{k} \frac{p_{k}\left(\frac{\alpha_{k}}{\varphi_{k}} \tau, \frac{z_{k}}{\varphi_{k}} e\right)}{T_{k}\left(\frac{\alpha_{k}}{\varphi_{k}} \tau, \frac{z_{k}}{\varphi_{k}} e\right)}, \quad \frac{1}{T\left(1 / \tau, e, \varphi_{A}, \alpha_{A}, z_{A}\right)}=\sum_{k \in \mathbb{K}} \frac{z_{k}}{T_{k}\left(\frac{\alpha_{k}}{\varphi_{k}} \tau, \frac{z_{k}}{\varphi_{k}} e\right)},
$$

under the constraints (48), (49) and (50) on the fractions.

Weak entropy solutions of the system satisfy

$$
\partial_{t}(\rho s)+\partial_{x}(\rho u s) \geq 0
$$

As relaxation towards the equilibrium is infinitely fast, one recovers the equilibrium fractions which satisfy

$$
Y^{\mathrm{immi}}(\tau, e) \stackrel{\text { def }}{=} \operatorname{argmax}_{\left(\varphi_{A}, \alpha_{A}, z_{A}\right)} s\left(\tau, e, \varphi_{A}, \alpha_{A}, z_{A}\right) .
$$


As a result the equilibrium pressure is

$$
p^{\mathrm{immi}}(\tau, e)=p\left(\tau, e, Y^{\mathrm{immi}}(\tau, e)\right) .
$$

We now turn to the definition of the relaxation source terms involved in the fraction equations in the system (73). Actually there is no consensus on their definition $[7,8]$. But they must relax towards the correct thermodynamic equilibrium and comply with the entropy growth criterion (76). Following for instance [3, 12, $13,16]$, one may choose $Q(Y) \stackrel{\text { def }}{=} \frac{\rho}{\lambda}\left(Y^{\text {immi }}(\tau, e)-Y\right)$, where $\lambda>0$ stands for a time relaxation. Thus the source term $Q$ is proportional to a density divided by a time. Hence one has

$$
\begin{aligned}
\partial_{t}(\rho s)+\partial_{x}(\rho u s) & =\nabla_{Y} s \cdot\left(\partial_{t} Y+u \partial_{x} Y\right) \\
& =\frac{\rho}{\lambda} \nabla_{Y} s \cdot\left(Y^{\mathrm{immi}}(\tau, e)-Y\right) \\
& \geq \frac{\rho}{\lambda}\left(s\left(\tau, e, Y^{\mathrm{immi}}\right)-s\left(\tau, e, \varphi_{A}, \alpha_{A}, z_{A}\right)\right) \geq 0,
\end{aligned}
$$

by the concavity of the mixture entropy $s$. This choice is then in agreement with the entropy growth criterion.

\subsection{Miscible case}

In the miscible case, the volume fractions are equal to one, see equation (54). Then we consider the additional advection equations on the mass and energy fractions of the phase $A, \widetilde{Y} \stackrel{\text { def }}{=}\left(\varphi_{A}, z_{A}\right)$

$$
\partial_{t} \tilde{Y}+u \partial_{x} \tilde{Y}=0
$$

Combining with the conservation of total mass and adding the source terms to the fraction equations gives

$$
\left\{\begin{array}{l}
\partial_{t}(\rho \widetilde{Y})+\partial_{x}(\rho u \widetilde{Y})=\widetilde{Q} \\
\partial_{t} \rho+\partial_{x}(\rho u)=0 \\
\partial_{t}(\rho u)+\partial_{x}\left(\rho u^{2}+\widetilde{p}\right)=0 \\
\partial_{t}(\rho \mathcal{E})+\partial_{x}((\rho \mathcal{E}+\widetilde{p}) u)=0
\end{array}\right.
$$

with the closure law

$$
\widetilde{p}=\widetilde{p}\left(1 / \rho, e, \varphi_{A}, z_{A}\right)
$$

The system (80) admits the concave function

$$
\widetilde{s}:\left(\tau, e, \varphi_{A}, z_{A}\right) \mapsto \varphi_{A} s_{A}\left(\frac{\tau}{\varphi_{A}}, \frac{z_{A}}{\varphi_{A}} e\right)+\varphi_{B} s_{B}\left(\frac{\tau}{1-\varphi_{A}}, \frac{1-z_{A}}{1-\varphi_{A}} e\right),
$$

as an entropy function, which complies with

$$
\mathrm{d} \widetilde{s}=\sum_{k \in \mathbb{K}} \frac{z_{k}}{T_{k}} \mathrm{~d} e+\sum_{k \in \mathbb{K}} \frac{p_{k}}{T_{k}} \mathrm{~d} \tau+\sum_{k \in \mathbb{K}} \partial_{\varphi_{k}} s \mathrm{~d} \varphi_{k}+\sum_{k \in \mathbb{K}} \partial_{z_{k}} s \mathrm{~d} z_{k} .
$$

We can then identify the mixture pressure (81) and the mixture temperature by

$$
\frac{\widetilde{p}\left(1 / \tau, e, \varphi_{A}, z_{A}\right)}{\widetilde{T}\left(1 / \tau, e, \varphi_{A}, z_{A}\right)}=\sum_{k \in \mathbb{K}} \frac{p_{k}\left(\frac{\tau}{\varphi_{k}}, \frac{z_{k}}{\varphi_{k}} e\right)}{T_{k}\left(\frac{\tau}{\varphi_{k}}, \frac{z_{k}}{\varphi_{k}} e\right)}, \quad \frac{1}{\widetilde{T}\left(1 / \tau, e, \varphi_{A}, z_{A}\right)}=\sum_{k \in \mathbb{K}} \frac{z_{k}}{T_{k}\left(\frac{\tau}{\varphi_{k}}, \frac{z_{k}}{\varphi_{k}} e\right)},
$$


under the constraints (48), (49) and (54) on the fractions.

Weak entropy solutions of the system satisfy

$$
\partial_{t}(\rho \widetilde{s})+\partial_{x}(\rho u \widetilde{s}) \geq 0
$$

As relaxation towards the equilibrium is infinitely fast, one recovers the equilibrium fractions which satisfy

$$
\widetilde{Y}^{\mathrm{mi}}(\tau, e) \stackrel{\text { def }}{=} \operatorname{argmax}_{\left(\varphi_{A}, z_{A}\right)} \widetilde{s}\left(\tau, e, \varphi_{A}, z_{A}\right)
$$

As a result the equilibrium pressure is

$$
\widetilde{p}^{\mathrm{mi}}(\tau, e)=\widetilde{p}\left(\tau, e, \widetilde{Y}^{\mathrm{mi}}(\tau, e)\right)
$$

Like in the immiscible case, a possible choice for the relaxation source terms on the fractions is $\widetilde{Q}(\widetilde{Y})=$ $\frac{\rho}{\lambda}\left(\widetilde{Y}^{\mathrm{mi}}(\tau, e)-\tilde{Y}\right)$, with $\lambda$ a positive timescale, which guarantees the entropy growth criterion thanks to the concavity of the mixture entropy $\widetilde{s}$.

\section{Conclusion \& Perspectives}

We address in this paper the construction of admissible equations of state for two-phase flows. Considering the mixture as a single fluid, it is possible to construct a complete EoS based on the knowledge of an incomplete EoS. This construction allows for instance to build the van der Waals entropy function starting from the pressure law $(\tau, T) \mapsto p$. However this law does not comply with convexity constraints required by standard Thermodynamics. Another construction consists in defining the mixture equilibrium entropy as an optimization problem on the phasic entropies. Depending on the mixture is immiscible or not, the mixture entropy corresponds to the concave hull of the phasic entropies or to their sup-convolution. Both operations guarantee concavity properties and, as a consequence, hyperbolicity of the multicomponent Euler system. However the mixture pressure laws are often difficult to compute and are, at best, tabulated EoS, which are difficult to manipulate and CPU consuming. One comes back to the remaining question 4 of the Introduction: "How to balance accuracy and reasonable computing times?" A solution is to consider rather HRM models. Hence the relaxation source terms have to relax towards the correct thermodynamic equilibrium while complying with the entropy growth criterion.

\section{REFERENCES}

[1] G. Allaire, G. Faccanoni, and S. Kokh. A strictly hyperbolic equilibrium phase transition model. C. R. Math. Acad. Sci. Paris, $344(2): 135-140,2007$.

[2] M. R. Baer and J. W. Nunziato. A two phase mixture theory for the deflagration to detonation (ddt) transition in reactive granular materials. Int. J. Multiphase Flow, 12(6):861-889, 1986.

[3] T. Barberon and P. Helluy. Finite volume simulation of cavitating flows. Computers and Fluids, 34(7):832-858, 2005.

[4] H. B. Callen. Thermodynamics and an Introduction to Thermostatistics. John Wiley \& sons, second edition, 1985.

[5] A. Chiapolino and R. Saurel. Extended noble-abel stiffened-gas equation of state for sub-and-supercritical liquid-gas systems far from the critical point. Fluids, 3:48, 072018.

[6] S. Dellacherie. Relaxation schemes for the multicomponent Euler system. M2AN Math. Model. Numer. Anal., 37(6):909-936, 2003.

[7] D. A. Drew and S. L. Passman. Theory of multicomponent fluids, volume 135 of Applied Mathematical Sciences. SpringerVerlag, New York, 1999.

[8] D.A. Drew. Mathematical modeling of two-phase flow. Ann. Rev. Fluid Mech., 15:261-291, 1983.

[9] L. C. Evans. Entropy and partial differential equations. Lecture Notes at UC Berkeley, 2004.

[10] L. C. Evans. A survey of entropy methods for partial differential equations. Bull. Amer. Math. Soc. (N.S.), 41(4):409-438, 2004.

[11] G. Faccanoni. Étude d'un modèle fin de changement de phase liquide-vapeur. Contribution à l'étude de la crise d'ébullition. PhD thesis, École Polytechnique, France, November 2008. 
[12] G. Faccanoni, S. Kokh, and G. Allaire. Modelling and simulation of liquid-vapor phase transition in compressible flows based on thermodynamical equilibrium. ESAIM Math. Model. Numer. Anal., 46(5):1029-1054, 2012.

[13] P. Helluy, O. Hurisse, and E. Le Coupanec. Verification of a two-phase flow code based on an homogeneous model. Int. J. Finite Vol., EDF Special Workshop:24, 2015.

[14] P. Helluy and J. Jung. Interpolated pressure laws in two-fluid simulations and hyperbolicity. In Finite volumes for complex applications. VII. Methods and theoretical aspects, volume 77 of Springer Proc. Math. Stat., pages 37-53. Springer, Cham, 2014.

[15] P. Helluy and H. Mathis. Pressure laws and fast Legendre transform. Math. Models Methods Appl. Sci., 21(4):745-775, 2011.

[16] P. Helluy and N. Seguin. Relaxation models of phase transition flows. M2AN Math. Model. Numer. Anal., 40(2):331-352, 2006.

[17] J.-M. Hérard. A class of compressible multiphase flow models. C. R. Math. Acad. Sci. Paris, 354(9):954-959, 2016.

[18] J.-B. Hiriart-Urruty and C. Lemaréchal. Fundamentals of convex analysis. Grundlehren Text Editions. Springer-Verlag, Berlin, 2001. Abridged version of it Convex analysis and minimization algorithms. I [Springer, Berlin, 1993; MR1261420 (95m:90001)] and it II [ibid.; MR1295240 (95m:90002)].

[19] O. Hurisse. Application of an homogeneous model to simulate the heating of two-phase flows. Int. J. Finite Vol., 11:37, 2014.

[20] O. Hurisse. Numerical simulations of steady and unsteady two-phase flows using a homogeneous model. Comput. E Fluids, 152:88-103, 2017.

[21] O. Hurisse and L. Quibel. A homogeneous model for compressible three-phase flows involving heat and mass transfer. 2018.

[22] S. Jaouen. Étude mathématique et numérique de stabilité pour des modèles hydrodynamiques avec transition de phase. PhD thesis, Pierre et Marie Curie University, Paris VI, France, 2001.

[23] J. Jung. Schémas numériques adaptés aux accélérateurs mutlicoeurs pour les écoulements bifluides. PhD thesis, Université de Strasbourg, 2013.

[24] F. Lagoutière. Modélisation mathématique et résolution numérique de problèmes de fluides compressibles à plusieurs constituants. PhD thesis, Pierre et Marie Curie University, Paris VI, France, 2000.

[25] L. Landau and E. Lifschitz. A Course of theoretical physics, vol 5, Statistical Physics, ch 8. Pergamon Press, 1969.

[26] O. Le Métayer, J. Massoni, and R. Saurel. Elaborating equations of state of a liquid and its vapor for two-phase flow models. Int. J. Therm. Sci., 43(3):265-276, 2004.

[27] O. Le Métayer and R. Saurel. The noble-abel stiffened-gas equation of state. Phys. Fluids, 28:046102, 2016.

[28] H. Mathis. Étude théorique et numérique des écoulements avec transition de phase. PhD thesis, Université Louis Pasteur, Strasbourg, 2010.

[29] H. Mathis. A thermodynamically consistent model of a liquid-vapor fluid with a gas. M2AN Math. Model. Numer. Anal., 2018.

[30] I. Müller and W. H. Müller. Fundamentals of thermodynamics and applications. Springer-Verlag, Berlin, 2009.

[31] R. T. Rockafellar. Convex analysis. Princeton Landmarks in Mathematics. Princeton University Press, Princeton, NJ, 1997. Reprint of the 1970 original, Princeton Paperbacks.

[32] R. H. Swendsen. An introduction to statistical mechanics and thermodynamics. Oxford University Press, 2012.

[33] N. W. Tschoegl. Fundamentals of equilibrium and steady-state thermodynamics. Elsevier, 2000.

\section{A. Legendre Transform and Maxwell Relations — intensive Description}

The entropy $s$ (or the energy $e$ ) are not directly physically measurable, whereas certain of the intensive variables (e.g. $T$ or $p$ ) are. It is consequently convenient to convert the entropy into functions of various intensive variables. Since $s$ is concave, we can employ ideas from convex analysis to rewrite various formula in terms of the intensive variables $T$ and $p$. The main tool will be de Legendre transform.

Assume that $f: \mathbb{R}^{n} \rightarrow(-\infty,+\infty]$ is a convex, lower semicontinuous (l.s.c.) and proper function $r \mapsto f(r)$. The Legendre transform of $f$ is the function $f^{*}: \mathbb{R}^{n} \rightarrow(-\infty,+\infty]$ defined by $f^{*}(q)=\sup _{r \in \mathbb{R}^{n}}(r \cdot q-f(r))$. The function $f^{*}$ is likewise convex, l.s.c. and proper. Furthermore $\left(f^{*}\right)^{*}=f$. Moreover, if $f$ is $\mathcal{C}^{2}$ and strictly convex then, given $q$, there exists a unique $r$ which maximizes $(r \cdot q-f(r))$, namely the unique $r=r(q)$ for which $q=\nabla f(r)$. In this case we have $f^{*}(q)=r(q) \cdot q-f(r(q))$ with $r=r(q)$ solving $q=\nabla f(r)$. Furthermore $\nabla f^{*}(q)=r+(q-\nabla f(r)) \nabla_{q} r=r$ and so $r=\nabla f^{*}(q)$.

In classic Thermodynamics it is convenient to define a complete EoS as the function $(s, \tau) \mapsto e$ instead of $(e, \tau) \mapsto s$ as in the present paper. Because the temperature positivity (8) holds, we can solve $s=s(e, \tau)$ and use $e$ as a $\mathcal{C}^{1}$ function of $(s, \tau)$, then we have $\mathrm{d} e=T \mathrm{~d} s-p \mathrm{~d} \tau$ and the Hessian matrix of $e$ is positive definite, 
that is

$$
\left.\left.\operatorname{det}\left(H_{e}\right) \stackrel{\text { def }}{=} \frac{\partial^{2} e}{\partial \tau^{2}}\right|_{s} \frac{\partial^{2} e}{\partial s^{2}}\right|_{\tau}-\left(\frac{\partial^{2} e}{\partial \tau \partial s}\right)^{2}>0,\left.\quad \frac{\partial^{2} e}{\partial \tau^{2}}\right|_{s}>0,\left.\quad \frac{\partial^{2} e}{\partial s^{2}}\right|_{\tau}>0
$$

It implies that the internal energy $e$ is strictly convex in $(s, \tau)$. There are three possible Legendre transforms of $(s, \tau) \mapsto e$, according to whether we transform: in the variable $s$ only, in $\tau$ only, or in $(s, \tau)$ together. Because it is customary in Thermodynamics to take the opposite of the mathematical Legendre transform (i.e. $-\sup \{\cdots\}=\inf \{-\cdots\})$, the relevant formula are actually the following:

- the Helmholtz free energy $(T, \tau) \mapsto f$ (sometimes noted $a$ ) is

$$
f(T, \tau)=\inf _{s}(e(s, \tau)-T s)=e(s, \tau)-T s
$$

where $s=s(T, \tau)$ solves $T=\left.\frac{\partial e}{\partial s}\right|_{\tau}$; the Helmholtz free energy $f$ is strictly concave in $T$, strictly convex in $\tau$;

- the enthalpy $(s, p) \mapsto h$ is

$$
h(s, p)=\inf _{\tau}(e(s, \tau)+p \tau)=e(s, \tau)+p \tau
$$

where $\tau=\tau(s, p)$ solves $-p=\left.\frac{\partial e}{\partial \tau}\right|_{s}$; the enthalpy $h$ is strictly concave in $p$, strictly convex in $s$;

- the Gibbs potential (a.k.a. chemical potential or free enthalpy) $(T, p) \mapsto \mu$ is

$$
\mu(T, p)=\inf _{(s, \tau)}(e(s, \tau)+p \tau-T s)=e(s, \tau)+p \tau-T s
$$

where $s=s(T, p)$ and $\tau=\tau(T, p)$ solve $T=\left.\frac{\partial e}{\partial s}\right|_{\tau}$ and $-p=\left.\frac{\partial e}{\partial \tau}\right|_{s}$; the Gibbs free energy $\mu$ is strictly concave in $(T, p)$.

These functions are called "Thermodynamic potentials". They are complete EoS describing the behaviour of a system as a function of the so-called "natural variables". In Table 1 we resume these relations. The Maxwell relations are obtained by exploiting the exactness of differentials (i.e. the order of the mixed second partial derivatives does not matter).

Using Legendre's transform of $(e, \tau) \mapsto s$, we can compute three other potentials (and associated Maxwell's relations, see Table 2) called Massieu-Planck Functions [32,33]:

- the function $\left(\frac{1}{T}, \tau\right) \mapsto j=\inf _{e}\left(s(e, \tau)-\frac{e}{T}\right)=s(e, \tau)-\frac{e}{T}$ where $e=e\left(\frac{1}{T}, \tau\right)$ solves $\frac{1}{T}=\left.\frac{\partial s}{\partial e}\right|_{\tau}$;

- the function $\left(e, \frac{p}{T}\right) \mapsto \star=\inf _{\tau}\left(s(e, \tau)-\frac{p}{T} \tau\right)=s(e, \tau)-\frac{p}{T} \tau$ where $\tau=\tau\left(e, \frac{p}{T}\right)$ solves $\frac{p}{T}=\left.\frac{\partial s}{\partial \tau}\right|_{e}$;

- the function $\left(\frac{1}{T}, \frac{p}{T}\right) \mapsto r=\inf _{(e, \tau)}\left(s(e, \tau)-\frac{p}{T} \tau-\frac{e}{T}\right)=s(e, \tau)-\frac{p}{T} \tau-\frac{e}{T}$ where $\tau=\tau\left(\frac{p}{T}, e\right)$ and $e=e\left(\frac{p}{T}, \frac{1}{T}\right)$ solve $\frac{p}{T}=\left.\frac{\partial s}{\partial \tau}\right|_{e}$ and $\frac{1}{T}=\left.\frac{\partial s}{\partial e}\right|_{\tau}$.

The function $j$ was first introduced by Massieu and it is called Massieu's potential. The function $r$ was introduced by Planck and is called Planck's potential.

Following similar ideas for $(\rho, \sigma) \mapsto \varepsilon$ and $(\rho, \varepsilon) \mapsto \sigma$ we obtain tables 3 and 4 . The function $\boldsymbol{\$}$ is sometimes called Kramer function [33, p.63].

The other functions are unnamed in our knowledge.

Note that, since $\varepsilon$ is strictly convex, its conjugate function is precisely $-p$, function of $(T, \mu)=\nabla \varepsilon(\sigma, \rho)$. Note that, since $\sigma$ is strictly concave, its conjugate function is precisely $\frac{p}{T}$, function of $\left(-\frac{\mu}{T}, \frac{1}{T}\right)=\nabla \sigma(\rho, \varepsilon)$. 
TABLE 1. Thermodynamic potentials based on complete $\operatorname{EoS}(s, \tau) \mapsto e$

\begin{tabular}{|c|c|c|c|c|c|}
\hline Potential & Natural & \multicolumn{2}{|c|}{ Conjugate variables } & Differential & Maxwell \\
\hline$e$ & $(s, \tau)$ & $T=\left.\frac{\partial e}{\partial s}\right|_{\tau}$ & $p=-\left.\frac{\partial e}{\partial \tau}\right|_{s}$ & $\mathrm{~d} e=T \mathrm{~d} s-p \mathrm{~d} \tau$ & $-\left.\frac{\partial p}{\partial s}\right|_{\tau}=\left.\frac{\partial T}{\partial \tau}\right|_{s}$ \\
\hline$f=e-T s$ & $(T, \tau)$ & $s=-\left.\frac{\partial f}{\partial T}\right|_{\tau}$ & $p=-\left.\frac{\partial f}{\partial \tau}\right|_{T}$ & $\mathrm{~d} f=-s \mathrm{~d} T-p \mathrm{~d} \tau$ & $\left.\frac{\partial p}{\partial T}\right|_{\tau}=\left.\frac{\partial s}{\partial \tau}\right|_{T}$ \\
\hline$h=e+p \tau$ & $(s, p)$ & $T=\left.\frac{\partial h}{\partial s}\right|_{p}$ & $\tau=\left.\frac{\partial h}{\partial p}\right|_{s}$ & $\mathrm{~d} h=T \mathrm{~d} s+\tau \mathrm{d} p$ & $\left.\frac{\partial \tau}{\partial s}\right|_{p}=\left.\frac{\partial T}{\partial p}\right|_{s}$ \\
\hline$\mu=e-T s+p \tau$ & $(T, p)$ & $s=-\left.\frac{\partial \mu}{\partial T}\right|_{p}$ & $\tau=\left.\frac{\partial \mu}{\partial p}\right|_{T}$ & $\mathrm{~d} \mu=-s \mathrm{~d} T+\tau \mathrm{d} p$ & $\left.\frac{\partial \tau}{\partial T}\right|_{p}=-\left.\frac{\partial s}{\partial p}\right|_{T}$ \\
\hline
\end{tabular}

TABlE 2. Thermodynamic potentials based on complete EoS $(e, \tau) \mapsto s$.

Note that $j=-f / T$ and $r=-\mu / T$

\begin{tabular}{|c|c|c|c|c|c|}
\hline Potential & $\begin{array}{c}\text { Natural } \\
\text { variables }\end{array}$ & \multicolumn{2}{|c|}{ Conjugate variables } & Differential & $\begin{array}{l}\text { Maxwell } \\
\text { relations }\end{array}$ \\
\hline$s$ & $(e, \tau)$ & $\frac{1}{T}=\left.\frac{\partial s}{\partial e}\right|_{\tau}$ & $\frac{p}{T}=\left.\frac{\partial s}{\partial \tau}\right|_{e}$ & $\mathrm{~d} s=\frac{1}{T} \mathrm{~d} e+\frac{p}{T} \mathrm{~d} \tau$ & $\left.\frac{\partial \frac{p}{T}}{\partial e}\right|_{\tau}=\left.\frac{\partial \frac{1}{T}}{\partial \tau}\right|_{e}$ \\
\hline$j=s-\frac{1}{T} e$ & $\left(\frac{1}{T}, \tau\right)$ & $e=-\left.\frac{\partial j}{\partial \frac{1}{T}}\right|_{\tau}$ & $\frac{p}{T}=\left.\frac{\partial j}{\partial \tau}\right|_{\frac{1}{T}}$ & $\mathrm{~d} j=-e \mathrm{~d} \frac{1}{T}+\frac{p}{T} \mathrm{~d} \tau$ & $\left.\frac{\partial \frac{p}{T}}{\partial \frac{1}{T}}\right|_{\tau}=-\left.\frac{\partial e}{\partial \tau}\right|_{\frac{1}{T}}$ \\
\hline$\star=s-\frac{p}{T} \tau$ & $\left(e, \frac{p}{T}\right)$ & $\frac{1}{T}=\left.\frac{\partial \star}{\partial e}\right|_{\frac{p}{T}}$ & $\tau=-\left.\frac{\partial \star}{\partial \frac{p}{T}}\right|_{e}$ & $\mathrm{~d} \star=\frac{1}{T} \mathrm{~d} e-\tau \mathrm{d} \frac{p}{T}$ & $-\left.\frac{\partial \tau}{\partial e}\right|_{\frac{p}{T}}=\left.\frac{\partial \frac{1}{T}}{\partial \frac{p}{T}}\right|_{e}$ \\
\hline$r=s-\frac{1}{T} e-\frac{p}{T} \tau$ & $\left(\frac{1}{T}, \frac{p}{T}\right)$ & $e=-\frac{\partial r}{\partial \frac{1}{T}} \mid$ & $\tau=-\left.\frac{\partial r}{\partial \frac{p}{T}}\right|_{\frac{1}{T}}$ & $\mathrm{~d} r=-e \mathrm{~d} \frac{1}{T}-\tau \mathrm{d} \frac{p}{T}$ & $\left.\frac{\partial \tau}{\partial \frac{1}{T}}\right|_{\frac{p}{T}}=\left.\frac{\partial e}{\partial \frac{p}{T}}\right|_{\frac{1}{T}}$ \\
\hline
\end{tabular}


TABLE 3. Thermodynamic potentials based on complete $\operatorname{EoS}(\sigma, \rho) \mapsto \varepsilon$.

Note that $\diamond=\rho f$ and $\varnothing \stackrel{(14)}{=}-p$

Potential

Natural

variables

Conjugate variables

Differential

Maxwell relations

\begin{tabular}{|c|c|c|c|c|c|}
\hline$\varepsilon$ & $(\sigma, \rho)$ & $T=\left.\frac{\partial \varepsilon}{\partial \sigma}\right|_{\rho}$ & $\mu=\left.\frac{\partial \varepsilon}{\partial \rho}\right|_{\sigma}$ & $\mathrm{d} \varepsilon=T \mathrm{~d} \sigma+\mu \mathrm{d} \rho$ & $\left.\frac{\partial \mu}{\partial \sigma}\right|_{\rho}=\left.\frac{\partial T}{\partial \rho}\right|_{\sigma}$ \\
\hline$\diamond=\varepsilon-T \sigma$ & $(T, \rho)$ & $\sigma=-\left.\frac{\partial \diamond}{\partial T}\right|_{\rho}$ & $\mu=\left.\frac{\partial \diamond}{\partial \rho}\right|_{T}$ & $\mathrm{~d} \diamond=-\sigma \mathrm{d} T+\mu \mathrm{d} \rho$ & $\left.\frac{\partial \mu}{\partial T}\right|_{\rho}=-\left.\frac{\partial \sigma}{\partial \rho}\right|_{T}$ \\
\hline$\nabla=\varepsilon-\mu \rho$ & $(\sigma, \mu)$ & $T=\left.\frac{\partial \nabla}{\partial \sigma}\right|_{\mu}$ & $\rho=-\left.\frac{\partial \nabla}{\partial \mu}\right|_{\sigma}$ & $\mathrm{d} \nabla=T \mathrm{~d} \sigma-\rho \mathrm{d} \mu$ & $-\left.\frac{\partial \rho}{\partial \sigma}\right|_{\mu}=\left.\frac{\partial T}{\partial \mu}\right|_{\sigma}$ \\
\hline$\oslash=\varepsilon-T \sigma-\mu \rho$ & $(T, \mu)$ & $\sigma=-\frac{\partial \odot}{\partial T} \mid$ & $\rho=-\left.\frac{\partial \odot}{\partial \mu}\right|_{T}$ & $\mathrm{~d} \varnothing=-\sigma \mathrm{d} T-\rho \mathrm{d} \mu$ & $\left.\frac{\partial \rho}{\partial T}\right|_{\mu}=\left.\frac{\partial \sigma}{\partial \mu}\right|_{T}$ \\
\hline
\end{tabular}

TABLE 4. Thermodynamic potentials based on complete $\operatorname{EoS}(\varepsilon, \rho) \mapsto \sigma$.

Note that $=\rho j$ and $\varnothing=-T \boldsymbol{h}$, that is $\boldsymbol{p}=-p / T$

\begin{tabular}{|c|c|c|c|c|c|}
\hline Potential & $\begin{array}{c}\text { Natural } \\
\text { variables }\end{array}$ & \multicolumn{2}{|c|}{ Conjugate variables } & Differential & $\begin{array}{l}\text { Maxwell } \\
\text { relations }\end{array}$ \\
\hline$\sigma$ & $(\varepsilon, \rho)$ & $\frac{1}{T}=\left.\frac{\partial \sigma}{\partial \varepsilon}\right|_{\rho}$ & $\frac{\mu}{T}=-\left.\frac{\partial \sigma}{\partial \rho}\right|_{\varepsilon}$ & $\mathrm{d} \sigma=\frac{1}{T} \mathrm{~d} \varepsilon-\frac{\mu}{T} \mathrm{~d} \rho$ & $-\left.\frac{\partial \frac{\mu}{T}}{\partial \varepsilon}\right|_{\rho}=\left.\frac{\partial \frac{1}{T}}{\partial \rho}\right|_{\varepsilon}$ \\
\hline$\diamond=\sigma-\frac{1}{T} \varepsilon$ & $\left(\frac{1}{T}, \rho\right)$ & $\varepsilon=-\left.\frac{\partial \downarrow}{\partial \frac{1}{T}}\right|_{\rho}$ & $\frac{\mu}{T}=-\left.\frac{\partial}{\partial \rho}\right|_{\frac{1}{T}}$ & $\mathrm{~d} \downarrow=-\varepsilon \mathrm{d} \frac{1}{T}-\frac{\mu}{T} \mathrm{~d} \rho$ & $\left.\frac{\partial \frac{\mu}{T}}{\partial \frac{1}{T}}\right|_{\rho}=\left.\frac{\partial \varepsilon}{\partial \rho}\right|_{\frac{1}{T}}$ \\
\hline $\boldsymbol{\phi}=\sigma+\frac{\mu}{T} \rho$ & $\left(\varepsilon, \frac{\mu}{T}\right)$ & $\frac{1}{T}=\left.\frac{\partial \mathbf{\varphi}}{\partial \varepsilon}\right|_{\frac{\mu}{T}}$ & $\rho=\left.\frac{\partial \mathbf{\varphi}}{\partial \frac{\mu}{T}}\right|_{\varepsilon}$ & $\mathrm{d} \boldsymbol{\varphi}=\frac{1}{T} \mathrm{~d} \varepsilon+\rho \mathrm{d} \frac{\mu}{T}$ & $\left.\frac{\partial \rho}{\partial \varepsilon}\right|_{\frac{\mu}{T}}=\left.\frac{\partial \frac{1}{T}}{\partial \frac{\mu}{T}}\right|_{\varepsilon}$ \\
\hline $\boldsymbol{q}=\sigma-\frac{1}{T} \varepsilon+\frac{\mu}{T} \rho$ & $\left(\frac{1}{T}, \frac{\mu}{T}\right)$ & $\varepsilon=-\frac{\partial \mathbf{p}}{\partial \frac{1}{T}} \mid$ & $\rho=\frac{\partial \hat{\mathbf{s}}}{\partial \frac{\mu}{T}} \mid$ & $\mathrm{d} \boldsymbol{\mu}=-\varepsilon \mathrm{d} \frac{1}{T}+\rho \mathrm{d} \frac{\mu}{T}$ & $\left.\frac{\partial \rho}{\partial \frac{1}{T}}\right|_{\frac{\mu}{T}}=-\frac{\partial \varepsilon}{\partial \frac{\mu}{T}} \mid$ \\
\hline
\end{tabular}

